\title{
Atrial standstill in a donkey with digitoxin intoxication
}

\author{
Antonia Ertelt', Christof A. Bertram², Friederike Stumpff', Selina Kuban', Robert Klopfleisch², Dr. Antina Lübke-Becker ${ }^{4}$ and Heidrun Gehlen' \\ 1 Klinik für Pferde, allgemeine Radiologie und Chirurgie, Freie Universität Berlin, Berlin, Germany \\ 2 Institut für Tierpathologie, Freie Universität Berlin, Berlin, Germany \\ 3 Institut für Veterinär-Physiologie, Freie Universität Berlin, Berlin, Germany \\ ${ }^{4}$ Institut für Mikrobiologie und Tierseuchen, Freie Universität Berlin, Berlin, Germany
}

Summary: A 15-year-old donkey gelding was referred to the Equine Clinic of the Freie Universität Berlin because of acute onset of anorexia, bradycardia and multiple syncopes. The clinical examination revealed an irregular heartbeat (11-18bpm) and syncopes. A continuous ECG revealed absence of P-waves and an irregular ventricular escape rhythm. An echocardiography revealed a pericardial effusion with reduction of left and right ventricular function. Laboratory abnormalities revealed increased troponin I, alpha hydroxybutyrate dehydrogenase and symmetric as well as asymmetric dimethylarginine. Increased concentrations of the cardiac glycoside digitoxin was evident in serum. Clinical findings were consistent with myocarditis with pericardial effusion and atrial standstill. Because of increasing severity of symptoms and grave prognosis the donkey was humanely euthanized. Histopathological examination of the cardiac atria including the sinoatrial node revealed severe, subacute, diffuse, suppurative myocarditis.

Keywords: atrial standstill, digitoxin, cardiac glycosides, myocarditis, troponin I, SDMA, ADMA, bradycardia, cardiology, donkey

Citation: Ertelt A., Bertram C. A., Stumpf F., Kuban S., Klopfleisch R., Lübke-Becker A., Gehlen H. (2020) Atrial standstill in a donkey with digitoxin intoxication. Pferdeheilkunde 36, 114-118; DOI 10.21836/PEM20200203

Correspondence: Dr. Antonia Ertelt, Freie Universität Berlin, Klinik für Pferde, Berlin, Oertzenweg 19b, 14193 Berlin, Germany; antonia.ertelt@fu-berlin.de

Submitted: December 2, 2019 | Accepted: December 30, 2019

A 15 -year-old, $253 \mathrm{~kg}$ donkey gelding was referred to the Equine Clinic of the Freie Universität Berlin to evaluate suspected colic and acute onset of bradycardia with multiple syncopes a few hours previously. Initial treatment by the referring veterinarian consisted of $15 \mathrm{ml}$ scopolamin butylbromid in combination with metamizole (buscopan compositum ${ }^{\circledR}$ ). The donkey was immediately referred to the Equine Clinic.

Abnormalities identified on physical examination included severe bradycardia (11-18 beats per minute (reference range 32-44) (Garba et al. 2015), tachypnea (44 breaths per min-

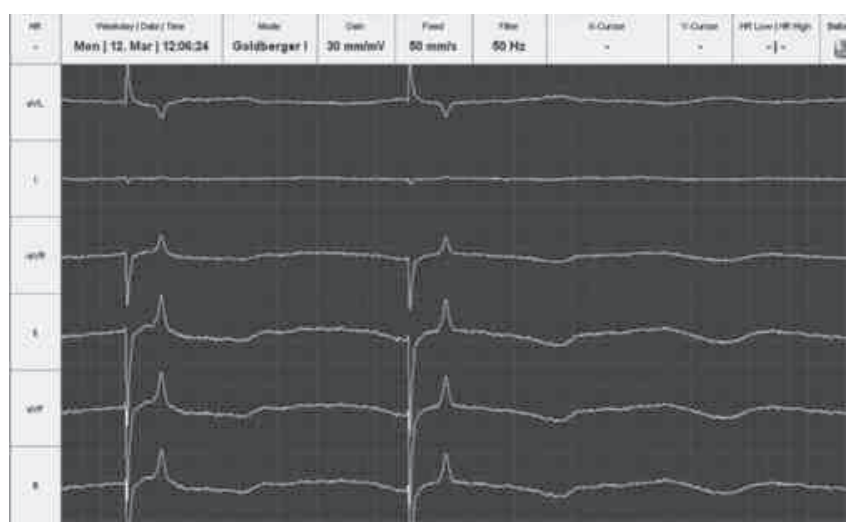

Fig. 1 A base-apex Holter ECG revealed absence of P-waves, irregular RR intervals, ST depression, positive T-waves and a variable ventricular escape rhythm at a rate of $11 \mathrm{bpm}$ (RR interval 5,25s). ute, reference 13-25) (Garba et al. 2015), tremor, poor pulse quality, decreased jugular refill and peripheral hypoperfusion (capillary refill time 4-5 seconds, cold extremities, ears and muzzle). Cardiac auscultation showed an irregular rhythm with a heart rate ranging between 11 to $18 \mathrm{bpm}$. A base-apex Holter ECG (Telvet 100) with four electrodes was performed.

The ECG revealed absence of $\mathrm{P}$-waves, and a variable ventricular escape rhythm at a rate of $11 \mathrm{bpm}$. The $S$ waves were negative, with positive $T$ waves. The RR-intervals were irregular, and ST-depression was obvious (figure 2). In the beginning, the morphology of each QRS-complex had an appear-

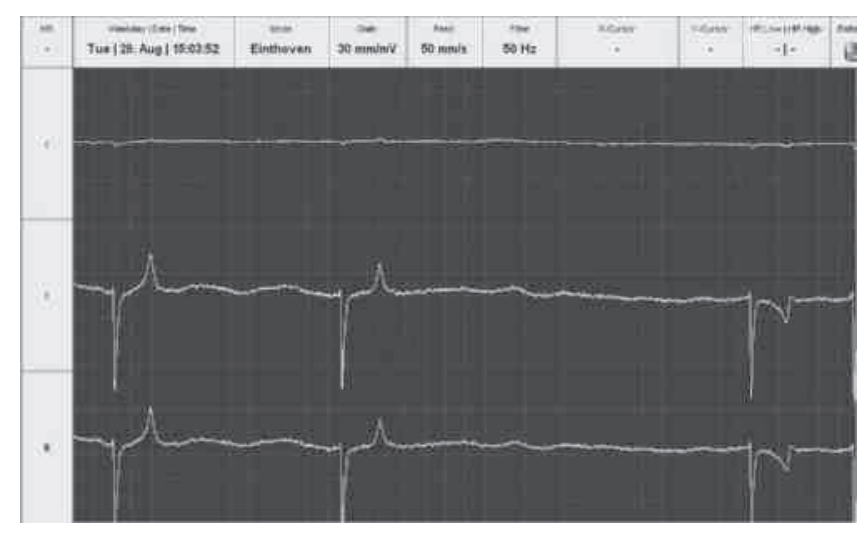

Fig. 2 QRS complexes were increased with amplitudes of $2,5 \mathrm{mV}$ in lead II (reference range horse $<2.2$ in lead II). 
ance normal for equines (figure 1). Later, the amplitude of the QRS-complex increased to $2.5 \mathrm{mV}$ in lead II (reference range horse $<2.2$ in lead II) (figure 2). The donkey collapsed during ECG recording when the heart rate dropped below $13 \mathrm{bpm}$ every 15 to 20 minutes. With disease progression, the T-waves became negative (figure 2), the RR-interval shortened (382 $\mathrm{ms}$ ) and the $\mathrm{S}$-wave amplitude became highly variable (1 mV-2.5 mV) (figure 3).

Echocardiographic examination (Vivid 7) revealed a pericardial effusion (figure 4). Left and right ventricular function were decreased (table 1). The atria showed no contractility. Color flow echocardiography revealed no relevant valve regurgitation.

Clinical findings were consistent with atrial standstill with a ventricular escape rhythm resulting in syncopes. Laboratory abnormalities included hemoconcentration, leukocytosis and neutrophilia (table 2). Furthermore, hyperglycemia and hyperlactatemia were obvious (table 2). The arterial blood gas analysis revealed hypercapnia and hypoxia (table 2). The donkey suffered from mixed acidosis (table 1). An increase in gamma-glutamyl transferase, blood urea nitrogen, creatinine, aspartate aminotransferase, lactate dehydrogenase and triglyceride were also present (table 2). Vitamin E (tocopherol) and selenium were within the reference range (table 2). Troponin I, alpha hydroxybutyrate dehydrogenase and symmetric as well as asymmetric dimethylarginine were increased (table $2)$. The catecholamine metabolites metanephrine and normetanephrine were severely increased (table 2). Despite the assertion that no glycosides had been given, assessment for digitoxin (but not for digoxin) was positive in serum (table 2).

Upon clinical admission, the donkey was suspected to suffer from severe myocarditis with pericardial effusion. Treatment with nasal oxygen insufflation, fluid administration (ringer lactate solution $80 \mathrm{ml} / \mathrm{kg}$, hypertonic saline $7.2 \% 4 \mathrm{mg} / \mathrm{kg}$ ), a parasympatholytic agent (atropine $0,01 \mathrm{mg} / \mathrm{kg} \mathrm{IV),} \mathrm{NSAID} \mathrm{(flunixin} 1.1 \mathrm{mg} /$ $\mathrm{kg} \mathrm{IV)} \mathrm{and} \mathrm{broad-spectrum} \mathrm{antibiotics} \mathrm{(amoxicillin} 10 \mathrm{mg} / \mathrm{kg}$ IV and gentamicin $2.2 \mathrm{mg} / \mathrm{kg} \mathrm{IV)} \mathrm{was} \mathrm{initiated.} \mathrm{The} \mathrm{developing}$ tachycardia was treated with lidocaine $(1.3 \mathrm{mg} / \mathrm{kg}$ bolus, CRI: $0,05 \mathrm{mg} / \mathrm{kg} / \mathrm{min}$ ). Because of increasing severity of symptoms and grave prognosis the donkey was humanely euthanized.

At a subsequent post-mortem examination, the pericardium was filled with approximately $400 \mathrm{ml}$ of a clear, serous fluid. The

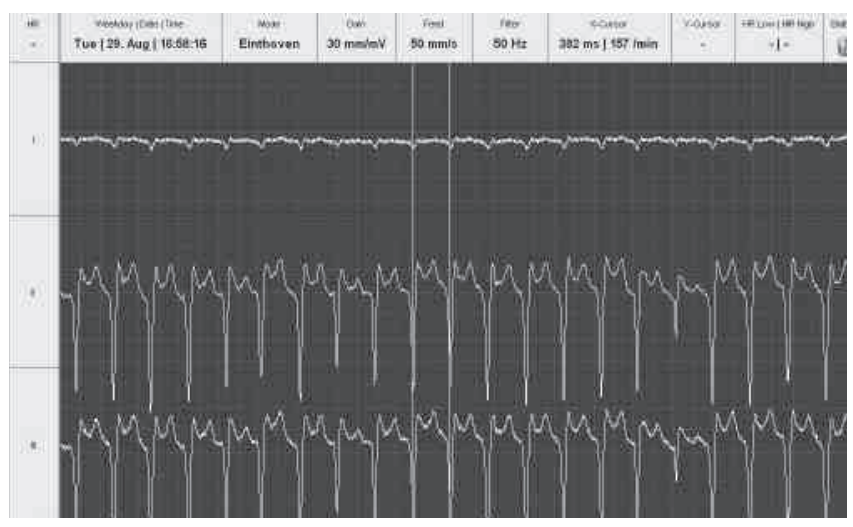

Fig. 3 With progression of the disease, the base-apex Holter ECG showed a further shortening of the RR-interval (382 ms, HR 157 bpm). A variable QRS amplitude (1 mV-2.5 mV) was noticeable. right ventricle was mildly dilated. There were multifocal, subendocardial petechia in the ventricle and atria. Sampling of the heart for histopathology was performed as described by Diab et al. 2017. The recommended 11 sections of the heart were routinely sectioned and stained with hematoxylin and eosin (H\&E) as well as azan for detection of collagen fibers. Histopathology of all sections including the right and left atrium (i.e. sections 1, 3-6 and 9 Diab et al. 2017) revealed moderate to severe, subacute, diffuse, suppurative myocarditis with pronounced hyaline degeneration of cardiomyocytes, severe intramuscular edema and severe acute hemorrhage (figure 5). Also, there were moderately increased fibroblasts and collagen fibers multifocally between the cardiomyocytes (figure $6 \mathrm{~A}$ and B). Inflammatory and fibrotic changes included the region around the sinoatrial node and to a lesser degree the sinoatrial node itself (section 4, figure 7) (Diab et al. 2017). Although the atrioventricular node was not detected by histopathology, suppurative myocarditis was also found in the region around the atrioventricular node (section 9, figure 7) (Diab et al. 2017). Sections of the ventricular myocardium did not reveal any changes with the exception of the occasional detection of neutrophilic granulocytes in close proximity to the valve plane.

Furthermore, there was an ascites with approximately $900 \mathrm{ml}$ of a clear, serous fluid in the abdominal cavity. Histopathology of the kidneys revealed moderate dilation of the cortical tubuli and marked proximal tubular epithelial cell degeneration. Findings of the kidney are consistent with acute ischemic tu-

\begin{tabular}{|c|c|}
\hline \multicolumn{2}{|c|}{ Left and right ventricular function } \\
\hline LV AMM FS & $0.5 \mathrm{~cm}$ \\
\hline LVEDV & $304.85 \mathrm{ml}$ \\
\hline LVESV & $37.04 \mathrm{ml}$ \\
\hline LVEF & $87.84 \%$ \\
\hline LVST & $67.4 \mathrm{ml}$ \\
\hline Lvecc d ch & $1.1 \mathrm{~cm}$ \\
\hline Lvecc s ch & $1.2 \mathrm{~cm}$ \\
\hline $\mathrm{RV}$ area/LV area $\mathrm{d} 4 \mathrm{ch}$ & $0.8 \mathrm{~cm}^{2}$ \\
\hline $\mathrm{RV}$ area/LV area s $4 \mathrm{ch}$ & $0.7 \mathrm{~cm}^{2}$ \\
\hline RVID/LVIDdM ch & $0.3 \mathrm{~cm}$ \\
\hline RVID/LVIDsM ch & $0.25 \mathrm{~cm}$ \\
\hline RV area FAC 4ch & $0.7 \mathrm{~cm}^{2}$ \\
\hline RV FAC left & $0.66 \mathrm{~cm}^{2}$ \\
\hline
\end{tabular}

LV: left ventricle; AMM: anatomical motion-mode; FS: fractional shortening, LVEDV: left ventricular end-diastolic volume; LVESV: left ventricular end-systolic volume, Lveecc d ch: left ventricular end-diastolic eccentricity index at chordal level measured in short-axis view at chordal level; Lveecc s ch: left ventricular end-systolic eccentricity index at chordal level measured in short-axis view at chordal level; RV area/LV area d4ch: Ratio between end-diastolic right and left ventricular area measured in long axis four-chamber view; RV area/LV area s4ch: Ratio between end-systolic right and left ventricular area measured in long axis four-chamber view; RVID/LVIDdM ch: Ratio between end-diastolic right and left ventricular internal diameter measured in short axis-view at chordal level in M-mode; RVID/LVIDsM ch: Ratio between end-systolic right and left ventricular internal diameter measured in short axis-view at chordal level in M-mode; RV area FAC 4ch: right ventricular fractional area change measured in long axis four-chamber view calculated as (RV area d 4ch-RV area s4ch)/RV area $\mathrm{d} 4 \mathrm{ch}$ $\times 100$; RV area FAC left: right ventricular fractional area change measured in long axis left ventricular outflow tract view calculated as (RV area d left-RV area s left)/RV area d left $\times 100$ 
bular injury most likely due to increased renal interstitial pressure in conjunction with output failure. Additionally, hepatic lipidosis was observed.

The lung showed a pronounced, diffuse, congestive hyperemia and severe, acute, alveolar edema.

Unfortunately, microbiological examination from freshly isolated heart tissue was not performed. Therefore, DNA was extracted from formalin-fixed myocardial tissue using a commercial kit. Multiplex Real Time PCR according to Cordoni et al. 2015 as well as qualitative eubacterial PCR according to Nadkarni et al. 2002 yielded negative results.

\section{Discussion}

Atrial standstill has not been previously described in donkeys. By definition, atrial standstill is a transient or persistent arrhythmogenic condition characterized by the complete or partial absence of electrical and mechanical activity in the atria (De Clercq et al. 2012).

From the outset, no P waves were detectable in the ECG of the donkey. Screening for glycosides showed high serum levels of digitoxin, the source of which remained obscure. Post mortem, histopathological examination showed that extensive parts of the atria had been destroyed. Most likely, glycoside intoxication with resulting myocarditis contributed to the cardiac syndrome observed in the donkey.

Initially, the QRS complexes had a normal appearance. Ventricular contraction was triggered by an irregular escape

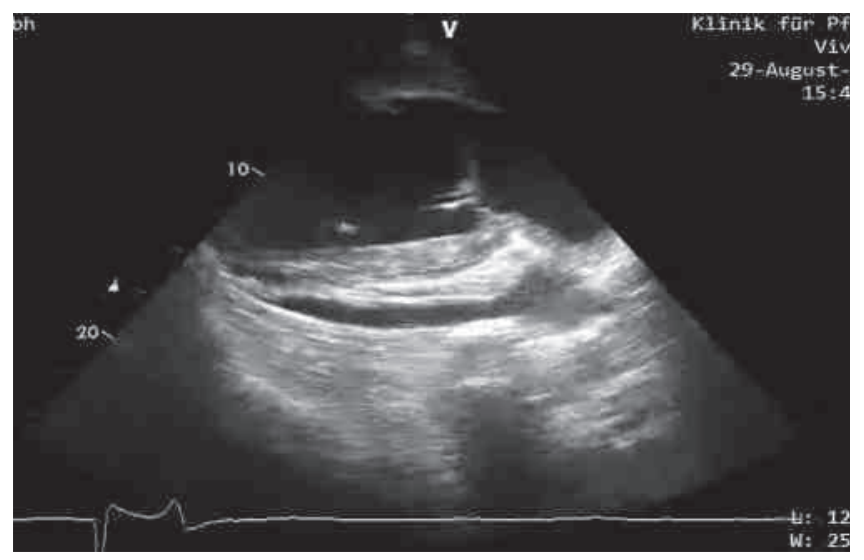

Fig. 4 Left parasternal long axis view with pericardial effusion. rhythm that was initially shaped as expected in horses, with a prominent negative $S$ wave.

A further pathological feature of the ECG was the T-wave inversion, most likely caused by toxic digitoxin levels (Table 1). However, similar effects are also seen in the second phase of pericarditis, reflecting defects in the repolarization of the tissue near the pericard. In line with this, T-wave inversion disappeared in later ECG recordings in the present case (figure 2 ), as to be expected deeper myocardial layers are affected in the course of progression from peri- to myocarditis. Ventricular ischemia may have further contributed to the ECG abnormalities observed (Hayden et al. 2002). This hypothesis is in line with the significant increase of troponin I and alpha hydroxybutyrate dehydrogenase as markers of myocarditis. Furthermore, a novel cardiac resp. renal biomarker that is being studied in our clinic - namely symmetric dimethylarginine - showed an elevation. Asymmetric dimethylarginine, a human cardiac biomarker, was also highly elevated.

Apart from myocarditis, causes for atrial standstill include glycoside intoxication and hyperkalemia (De Clercq et al. 2012). While systemic hyperkalemia was at no time present in the donkey, serum digitoxin was elevated. Since iatrogenic exposure of the donkey to digitoxin could not be verified despite scrutiny, it appears

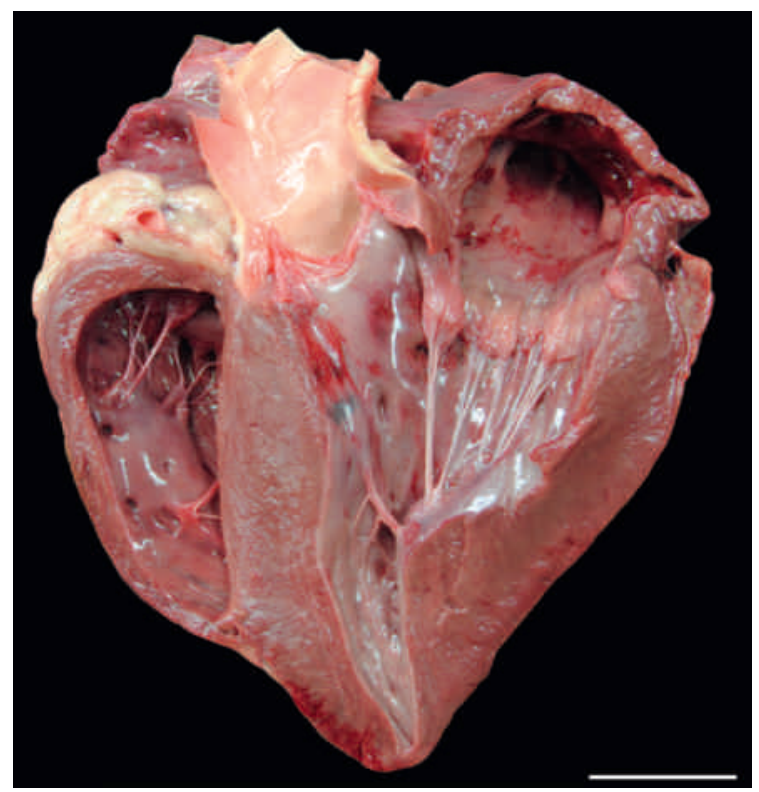

Fig. 5 Gross image of the longitudinal section of the heart with multifocal, subendocardial petechia in the ventricle and atria. Bar $=5 \mathrm{~cm}$

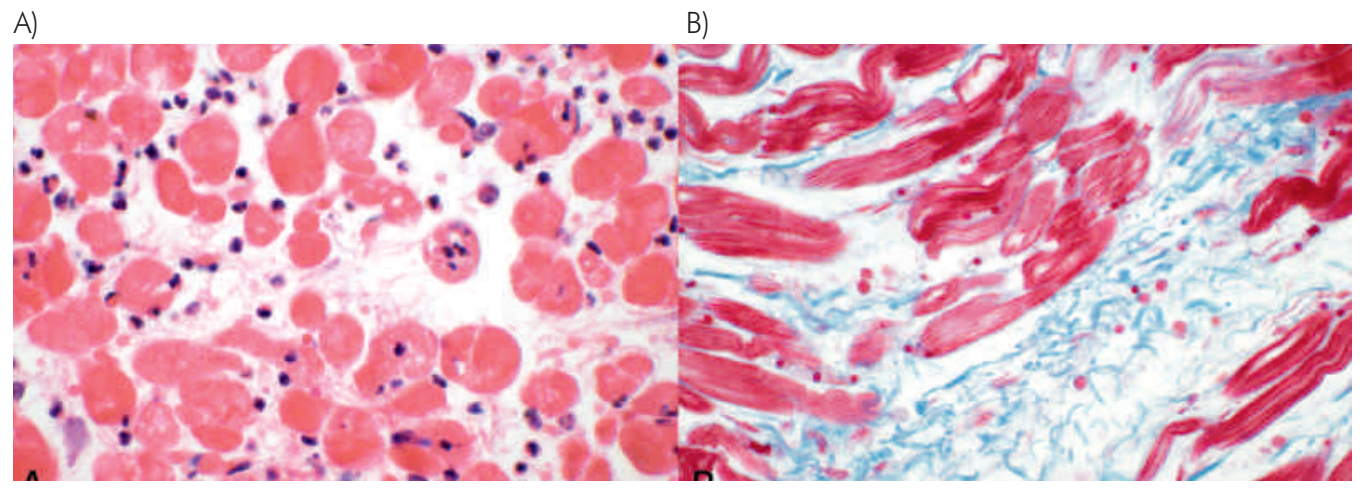

Fig. 6 A) Histopathological image of the atrial myocardium with moderate infiltration with neutrophilic granulocytes (purulent atrial myocarditis), hyaline degeneration of cardiomyocytes and intramuscular edema. H\&E stain. Bar $=20 \mu \mathrm{m}$. B) Histopathological image of the atrial myocardium with multifocal deposition of collagen fibers (blue). Azan stain. Bar $=20 \mu \mathrm{m}$ 
possible to speculate that the intoxication might have been the result of ingestion of glycoside containing plants (e.g., oleander,

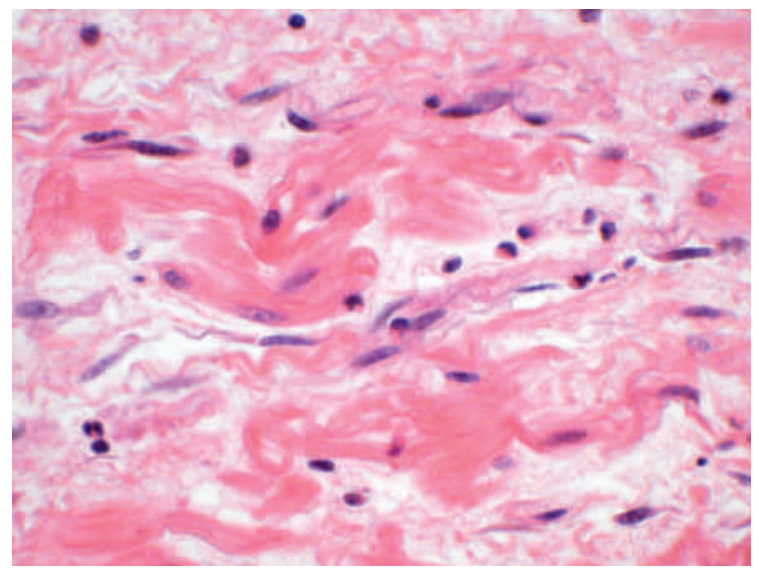

Fig. 7 Histopathological image of the sinoatrial node with mild infiltration with neutrophilic granulocytes. H\&E stain. Bar $=20 \mu \mathrm{m}$ rhododendron, foxglove, lily of the valley, etc.). Foxglove (digitalis) contains several cardioactive substances including digoxin, which is metabolized to digitoxin within the body. Foxglove intoxication gives a very distinct ECG pattern, including blocks, ventricular tachycardia, and ST-deviation, along with nephritis and colitis. However, the precursor digoxin was not measured in plasma and signs of colitis or findings of plant ingredients were absent in necropsy so that this possibility remains speculative.

Due to parasympathetic action, cardiac glycosides have direct negative chronotropic and dromotropic effects (De Clercq et al. 2012). Furthermore, cardiac glycosides induce rises in cytosolic $\mathrm{Ca}^{2+}$ which enhance contractility at physiological dosages but have been implicated in glycoside-induced apoptisis with necrosis and inflammation of the myocardium in cases of intoxication (Hayden et al. 2002, Galey et al. 1996). This might explain the histological finding of myocarditis in the current case. Apart from toxic insults, causes for myocarditis include viral or bacterial infections, parasitic migration, fungal infections, nutritional imbalances,

\begin{tabular}{|c|c|c|c|}
\hline Laboratory results & & & \\
\hline & measured & reference range & reference \\
\hline packed cell volume & $49 \%$ & $31-43 \%$ & donkey [ Garba et al. 2015] \\
\hline red blood cells & $9.2 \times 10^{12} / \mathrm{L}$ & $4.4-7 \times 10^{12} / \mathrm{L}$ & donkey [Burden et al. 2016] \\
\hline white blood cells & $14.92 \times 10^{9} / \mathrm{L}$ & $3.5-10.9 \times 10^{9} / \mathrm{L}$ & donkey [ Garba et al. 2015] \\
\hline neutrophile granulocytes & $12.9 \times 10^{9} / \mathrm{L}$ & $2.4-6.3 \times 10^{9} / \mathrm{L}$ & donkey [ Burden et al. 2016] \\
\hline glucose & $260 \mathrm{mg} / \mathrm{dL}$ & $69.1-82.9 \mathrm{mg} / \mathrm{dL}$ & donkey [ Garba et al. 2015] \\
\hline lactate & $11.9 \mathrm{mg} / \mathrm{dL}$ & $0.5-2.0 \mathrm{mg} / \mathrm{dL}$ & institutional reference range horse \\
\hline $\mathrm{PaO}_{2}$ & $52 \mathrm{mmHg}$ & $95-105 \mathrm{mmHg}$ & institutional reference range horse \\
\hline $\mathrm{PaCO}_{2}$ & $60 \mathrm{mmHg}$ & $35-45 \mathrm{mmHg}$ & institutional reference range horse \\
\hline $\mathrm{pH}$ & 7.28 & $7.34-7.44$ & institutional reference range horse \\
\hline bicarbonate & $19.5 \mathrm{mmol} / \mathrm{L}$ & $21.7-26.3 \mathrm{mmol} / \mathrm{L}$ & donkey [ Garba et al. 2015] \\
\hline anion gap & $29 \mathrm{mmol} / \mathrm{L}$ & $9-17 \mathrm{mmol} / \mathrm{L}$ & institutional reference range horse \\
\hline lactate dehydrogenase & $527 \mathrm{U} / \mathrm{L}$ & $>400 \mathrm{U} / \mathrm{L}$ & laboklin laboratory \\
\hline -glutamyl transferase & $197 \mathrm{U} / \mathrm{L}$ & $14-69 \mathrm{U} / \mathrm{L}$ & donkey [ Burden et al. 2016] \\
\hline aspartate aminotransferase & $584 \mathrm{U} / \mathrm{L}$ & $238-536 \mathrm{U} / \mathrm{L}$ & donkey [ Burden et al. 2016] \\
\hline creatinine & $6.38 \mathrm{mg} / \mathrm{dL}$ & $0.6-1.34 \mathrm{mg} / \mathrm{dL}$ & donkey [ Burden et al. 2016] \\
\hline blood urea nitrogen & $185 \mathrm{mg} / \mathrm{dL}$ & $27-93 \mathrm{mg} / \mathrm{dL}$ & donkey [ Burden et al. 2016] \\
\hline troponin I & $50 \mathrm{ng} / \mathrm{mL}$ & $>0.03 \mathrm{ng} / \mathrm{mL}$ & laboklin laboratory \\
\hline$\alpha$-hydroxybutyrate dehydrogenase & $290 U / L$ & $>170 \mathrm{U} / \mathrm{L}$ & laboklin laboratory \\
\hline $\mathrm{LDH} / \mathrm{HBDH}$ & $2: 1.1$ & $2: 1$ & laboklin laboratory \\
\hline atrial natriuretic peptide & $4558 \mathrm{pg} / \mathrm{mL}$ & $2023-22714 \mathrm{pg} / \mathrm{mL}$ & institutional reference range horse \\
\hline symmetric dimethylarginine & $2.1 \mu \mathrm{mol} / \mathrm{L}$ & $0.3-0.8 \mu \mathrm{mol} / \mathrm{L}$ & DLD diagnostica $\mathrm{GmbH}$ \\
\hline asymmetric dimethylarginine & $9.0 \mu \mathrm{mol} / \mathrm{L}$ & $1.7-3.8 \mu \mathrm{mol} / \mathrm{L}$ & DLD diagnostica $\mathrm{GmbH}$ \\
\hline metanephrine & $459 \mathrm{pg} / \mathrm{mL}$ & $9.3-186.1 \mathrm{pg} / \mathrm{mL}$ & institutional reference range horse \\
\hline normethanephrine & $585 \mathrm{pg} / \mathrm{mL}$ & $40.1-324.4 \mathrm{pg} / \mathrm{mL}$ & institutional reference range horse \\
\hline triglyceride & $430 \mathrm{mg} / \mathrm{dL}$ & $20.0-144.0 \mathrm{mg} / \mathrm{dL}$ & donkey [Dugat et al. 2010] \\
\hline vitamin $\mathrm{E}$ & $8.4 \mathrm{mg} / \mathrm{L}$ & $>1 \mathrm{mg} / \mathrm{L}$ & laboklin laboratory \\
\hline selen & $92 \mu \mathrm{g} / \mathrm{L}$ & $50-150 \mu \mathrm{g} / \mathrm{L}$ & laboklin laboratory \\
\hline digoxin & $<0.5 \mu \mathrm{g} / \mathrm{L}$ & $<0.5 \mu \mathrm{g} / \mathrm{L}$ & IDEXX laboratories \\
\hline digitoxin & $28.6 \mu \mathrm{g} / \mathrm{L}$ & $<0.5 \mu \mathrm{g} / \mathrm{L}$ & IDEXX laboratories \\
\hline
\end{tabular}

$\mathrm{PaO}_{2}$ : partial pressure of oxygen in arterial blood, $\mathrm{PaCO}_{2}$ : partial pressure of carbon dioxide in arterial blood, LDH: lactate dehydrogenase; $\mathrm{HBDH}$ : $\alpha$-hydroxybutyrate dehydrogenase 
hyperthermia, hypoxia, immune mediated and idiopathic disorders for which we had no evidence (van Loon 2012, Breuhaus 2011).

With ongoing disease, the donkey developed ventricular tachycardia. This change to ventricular tachyarrhythmia is a consequence of low output failure due to bradycardia and the progressive reduction in cardiac output, undoubtedly caused both by cardiac tamponade due to increasing pericardial fluid accumulation and direct myocardial damage. Hypoxemia can lead to local hyperkalemia (Carmeliet 1999), inducing hyperexcitability with ectopic generation of action potentials. Both hypoxia (Hammarstrom and Gage 2002) and concomitant acidosis (Jones et al. 2014) can interfere with the function of cardiac ion channels (Carmeliet 1999, Hammarstrom and Gage 2002, Jones et al. 2014), leading to increased excitability of cardiac tissues.

When digoxin toxicity is suspected, dioxin immune Fab fragments (antibodies) are given to human patients (Higgins et al. 1989). Furthermore, treatment with activated charcoal and laxatives via nasogastric tube may be beneficial to clear the intestine and prevent further absorption of glycosides.

Less specific but essential measures include management of acidosis and anticoagulant treatment. Patients with bradycardia due to atrial standstill can benefit from sympathomimetic agents or vagolytic agents, although sympathomimetics may enhance tissue excitability and promote reentry phenomena (McGuirk and Muir 1985, Bonagura et al. 2010, Davey 1986). For this reason, implantation of a pacemaker is usually preferable (van Loon et al. 2002, Pibarot et al. 1993, Reef et al. 1986). In the current case, emergency pericardiocentesis might have been tried, however, it is highly unlikely that either this or implantation of a pacemaker would have changed the prognosis of the severely ill patient.

\section{References}

Bonagura J. D., Reed M. S., Bayly W. M., Sellon D. C. (2010) Equine internal medicine. Third Edition, Sounders Elsvier 425-463

Breuhaus B. (2011) Equine cardiology - infectious, inflammatory, and neoplastic diseases (Proceedings). CVC In San Diego Proceedings

Burden F. A., Hazell-Smith E., Mulugeta G., Patrick V., Trawford R., Brooks Brownlie H. W. (2016) Reference intervals for biochemical and haematological parameters in mature domestic donkeys (Equus asinus) in the UK. Equine Vet. Educ. 28, 134-139; DOI 10.1111/eve.12512

Carmeliet E. (1999) Cardiac ionic currents and acute ischemia: from channels to arrhythmias. Physiol. Rev. 79, 917-1017; DOI 10.1152/physrev. 1999.79.3.917
Cordoni G., Williams A., Durham A., Florio D., Zanoni R. G., La Ragione R. M. (2015) Rapid diagnosis of strangles (Streptococcus equi subspecies equi) using PCR. Res. Vet. Sci. 102,162-166, DOI 10.1016/j.rvsc.2015.08.008

Davey M. J. (1986) Alpha adrenoceptors - an overview. J. Mol. Cell Cardiol. 5, 1-15; 10.1016/s0022-2828(86)80457-6

De Clercq D., Verheyen T., Decloedt A., Lefère L., van Loon G. (2012) Atrial standstill in a horse with cardiac glycoside intoxication. European Veterinary Conference Voorjaarsdagen, Abstracts 302

Diab S. S., Poppenga R., Uzal F. A. (2017) Sudden death in racehorses: post-mortem examination protocol. J. Vet. Diagn. Invest. 1-8

Dugat S. L., Taylor T. S., Matthews N. S., Gold J. R. (2010) Values for triglycerides, insulin, cortisol, and ACTH in a herd of normal donkeys. J. Equine Vet. Sci. 30, 141-144; DOI 10.1016/j.jevs.2010.01.054

Galey F. D., Holstege D. M., Plumlee K. H., Tor E., Johnson B., Anderson M. L., Blanchard P. C., Brown F. (1996) Diagnosis of oleander poisoning in livestock. J. Vet. Diagn. Invest. 8, 358-364; DOI $10.1177 / 104063879600800314$

Garba U. M., Sackey A. K. B., Idris L. A., Esievo K. A. N. (2015) Baseline vital, haematological and serum biochemical parameters of donkeys. J. Vet. Med. Ani. Health 7, 94-98; DOI 10.5897/JVMAH2014.0353

Hammarstrom A. K., Gage P. W. (2002) Hypoxia and persistent sodium current. Eur. Biophys. J. 31, 323-330; PMID 12202908, DOI 10.1007/s00249-002-0218-2

Hayden G. E., Brady W. J., Perron A. D., Somers M. P., Mattu A. (2002) Electrocardiographic T-wave inversion: differential diagnosis in the chest pain patient. Am. J. Emerg. Med. 20, 252-262

Higgins G. L., Campbell B. A. (1989) Digoxin-immune Fab fragments. Ann. Emerg. Med. 18, 113; PMID 2910151

Jones D. K., Ruben P. C. (2014) Proton modulation of cardiac I Na: a potential arrhythmogenic trigger. Handb. Exp. Pharmacol. $221169-81$; 10.1007/978-3-642-41588-3 8

McGuirk S. M., Muir W. W. (1985) Diagnosis and treatment of cardiac arrhythmias. Vet. Clin. North Am. Equine Pract. 1, 353-370; 10.1016/s0749-0739(17)30760-5

Nadkarni M. A., Martin F. E., Jacques N. A., Hunter N. (2002) Determination of bacterial load by real-time PCR using a broad-range (universal) probe and primers set. Microbiology 148, 257-266; DOI 10.1099/00221287-148-1-257

Pibarot P., Vrins A., Salmon Y. (1993) Implantation of a programmable atrioventricular pacemaker in a donkey with complete atrioventricular block and syncope. Equine Vet. J. 25, 248-251; DOI 10.1111/i.2042-3306.1993.tb02955.x

Reef V. B., Clark E. S., Oliver J. A. (1986) Implantation of a permanent transvenous pacing catheter in a horse with complete heart block and syncope. J. Am. Vet. Med. Assoc. 189, 449-452; PMID 3759616

van Loon G. (2012) Inflammatory myocardial disease and toxicity in horses - a recent update. Veterinary Cardiovascular Society Meeting. Loughborough, UK 1:3

van Loon G., Fonteyne W., Rottiers H., Tavernier R., Deprez P. (2002) Implantation of a dual-chamber, rate-adaptive pacemaker in a horse with suspected sick sinus syndrome. Vet. Rec. 151, 541-545; DOI 10.1111/j.1939-1676.2001.tb01592.x 